\title{
Multivariate Multiscale Entropy for Brain Consciousness Analysis
}

\author{
Mosabber Uddin Ahmed, Ling Li, Jianting Cao and Danilo P. Mandic
}

\begin{abstract}
The recently introduced multiscale entropy (MSE) method accounts for long range correlations over multiple time scales and can therefore reveal the complexity of biological signals. The existing MSE algorithm deals with scalar time series whereas multivariate time series are common in experimental and biological systems. To that cause, in this paper the MSE method is extended to the multivariate case. This allows us to gain a greater insight into the complexity of the underlying signal generating system, producing multifaceted and more robust estimates than standard single channel MSE. Simulations on both synthetic data and brain consciousness analysis support the approach.
\end{abstract}

\section{INTRODUCTION}

The Takens embedding theorem [1] implies that although the true dynamics of a complex system generating e.g. biological signal may be unknown, such dynamics may still be recovered using time delayed embedding of a single time series, seen as a one-dimensional projection of the multivariate system trajectory. This way we can estimate 'invariant quantities' of the original system, such as attractor dimensions, Lyapunov exponents and entropies [2][3], from the time delay embedded reconstructed dynamics. One such invariant measure (in terms of smooth transformations of the state space of the attractor) is the recently introduced multiscale entropy (MSE) [4], a method that computes sample entropy from single time series, coarse grained on multiple scales, and thus reveals long-range correlations over a range of temporal scales within a complex system.

It is important to notice that traditional entropy measures such as Shannon entropy [2], Kolmogorov-Sinai (KS) entropy [5], approximate entropy (ApEn) [6] and sample entropy (SampEn) [7] can be used to quantify only the regularity (predictability) of time series on a single scale, by e.g. evaluating the appearance of repetitive patterns [4]. However, there is no straightforward correspondence between regularity and signal complexity.

Neither completely predictable (e.g. periodic) signals nor completely unpredictable (e.g. uncorrelated random) signals are truly complex, since at a global level they admit a very simple description. As a result, the output of complex systems is far from the two extrema of perfect regularity and complete randomness. Instead, complex systems generally exhibit long-range correlations on multiple spatial

M. U. Ahmed, L. Li and D. P. Mandic are with the Department of Electrical and Electronic Engineering, Imperial College London, London SW7 2AZ, U.K. mosabber . ahmed@imperial . ac.uk

J. Cao is with the Department of Electronic Engineering, Saitama Institute of Technology, Japan, East China University of Science and Technology, Shanghai, 200237, China and also with the Laboratory for Advanced Brain Signal Processing, RIKEN Brain Science Institute, Saitama 351-0198, Japan caodsit.ac.jp and temporal scales. These multiscale features, ignored by conventional entropy calculations, are explicitly addressed by the MSE method [4].

Applications of MSE include the analysis of fluctuations of the human heartbeat under pathologic conditions like erratic cardiac arrhythmia and congestive heart failure [4], analysis of EEG and MEG recordings in patients with Alzheimer's disease [8], and insights into the complexity of human gait dynamics from healthy subjects under different conditions [9]. In addition, MSE has been used to examine variations in EEG complexity in response to photic stimulation during aging [10], to study complex dynamics of human red blood cell flickering and alterations with in vivo aging [11]. All the reported results strongly support the general 'complexity-loss' theory for systems under 'stress', for instance, through aging and disease [12].

The existing MSE algorithm has some shortcomings [13] and is limited to the analysis of scalar time series. However, multivariate time series are routinely measured in experimental and biological systems. Although multivariate time series can be treated as a set of individual time series by considering each variable separately, this is only efficient if all the variables are statistically independent or uncorrelated at the very least (which is often not the case). For example, measurements of the $\mathrm{z}$-coordinate of the Lorenz equations cannot reconstruct the dynamics of Lorenz system because they do not resolve the $\mathrm{x}-\mathrm{y}$ symmetry [14].

Although, in principle, by observing a scalar time series for a sufficiently long time enables us to reconstruct the dynamics of the underlying systems, in practice this is extremely difficult when more than a couple degrees of freedom are present (a common scenario for biological systems). There are substantial advantages in analysing several different time series in response to the same phenomenon if they are available, especially if the system is noisy (typical for real data). For instance, in medical diagnosis, in addition to the electrocardiogram (ECG), a medic would also look into the respiratory rate, blood pressure and electroencephalogram (EEG) to assess the health state of a patient. Thus, the increasing interest in reverse-engineering biological systems from the observed time series of typically multivariate, finite and noisy nature also motivates us to extend the univariate MSE to the multivariate case. We therefore first introduce multivariate sample entropy and use it to perform multivariate multiscale entropy (MMSE) analysis simultaneously for any number of data channels. The proposed method is supported by analysis on a real world example of distinguishing between the brain consciousness states of coma and quasibrain-death. 


\section{MULTIVARIATE MULTISCALE ENTROPY ANALYSIS}

\section{A. Multiscale Entropy Analysis}

In 2002, Costa et al. [4] proposed multiscale entropy (MSE) as a meaningful physiologic complexity measure which evaluates the relative complexity of normalized time series across multiple scales. The MSE methodology has two steps:

- Multiple coarse-grained time series are generated from the original time series $\left\{x_{1}, x_{2}, \ldots, x_{N}\right\}$ by averaging the data points within non-overlapping windows of increasing length $\epsilon$, also known as the scale factor. The elements of the coarse-grained time series of scale factor $\epsilon$ are calculated as:

$$
y_{j}^{\epsilon}=\frac{1}{\epsilon} \sum_{i=(j-1) \epsilon+1}^{j \epsilon} x_{i} \quad \text { where, } 1 \leq j \leq \frac{N}{\epsilon}
$$

The length of each coarse-grained series is $\epsilon$ times shorter than that of the original series. For scale 1, the coarse-grained time series is therefore equivalent to the original one.

- The sample entropy, SampEn [7], is calculated for each coarse-grained time series, and then plotted as a function of the scale factor.

Similarly, to introduce the multivariate multiscale entropy (MMSE) analysis method, we propose the following two steps:

- Coarse-grain the multivariate time series $\left\{x_{k, i}\right\}_{i=1}^{N}, k=$ $1,2, \ldots, p$ where $p$ denotes the number of variables and $N$ the number of samples in each variable. The elements of the multivariate coarse-grained time series of scale factor $\epsilon$ are calculated as:

$$
y_{k, j}^{\epsilon}=\frac{1}{\epsilon} \sum_{i=(j-1) \epsilon+1}^{j \epsilon} x_{k, i}
$$

where, $1 \leq j \leq \frac{N}{\epsilon}$ and $k=1$ to $p$.

- Calculate the multivariate sample entropy, MSampEn, for each multivariate coarse-grained time series, and plot it as a function of the scale factor.

\section{B. Multivariate Embedding}

To calculate multivariate sample entropy, we first need to generate multivariate embedded vectors. Cao et al. [14] were the first to generalize the Takens embedding theorem for multivariate case. Given that there are $p$ time series $\left\{x_{k, i}\right\}_{i=1}^{N}, k=1,2, \ldots, p$ generated through $p$ measurement functions $h_{k}\left(y_{i}\right)$ from the same system, the multivariate embedded reconstruction is given by:

$$
\begin{aligned}
X_{m}(i)= & {\left[x_{1, i}, \ldots, x_{1, i+\left(m_{1}-1\right) \tau_{1}}, x_{2, i}, \ldots,\right.} \\
& \left.x_{2, i+\left(m_{2}-1\right) \tau_{2}}, \ldots, x_{p, i}, \ldots, x_{p, i+\left(m_{p}-1\right) \tau_{p}}\right]
\end{aligned}
$$

where $M=\left[m_{1}, m_{2}, \ldots, m_{p}\right]$ is the embedding vector, $\boldsymbol{\tau}=$ $\left[\tau_{1}, \tau_{2}, \ldots, \tau_{p}\right]$ is the time lag vector and $m=\sum_{k=1}^{p} m_{k}$.

\section{Multivariate Sample Entropy}

Richman \& Moorman [7] introduced the sample entropy (SampEn) which represents the conditional probability that two sequences of $m$ consecutive data points, which are similar within a tolerance level $r$ will remain similar when the next consecutive point is included, provided that self-matches are not considered in calculating the probability. Before introducing the multivariate sample entropy, an issue of concern with multivariate data is that they are not guaranteed to have the same range, so that the distances calculated on embedded vectors may depend highly on the components of the variate with largest ranges. It is therefore often preferred to scale all the data to have either the same variance or to be in the same amplitude range. In our formulation of multivariate sample entropy, we opt to scale the data to the range $[0,1]$.

For a $p$-variate time series $\left\{x_{k, i}\right\}_{i=1}^{N}, k=1,2, \ldots, p$, the algorithm to calculate multivariate sample entropy (MSampEn) is given in Algorithm 1.

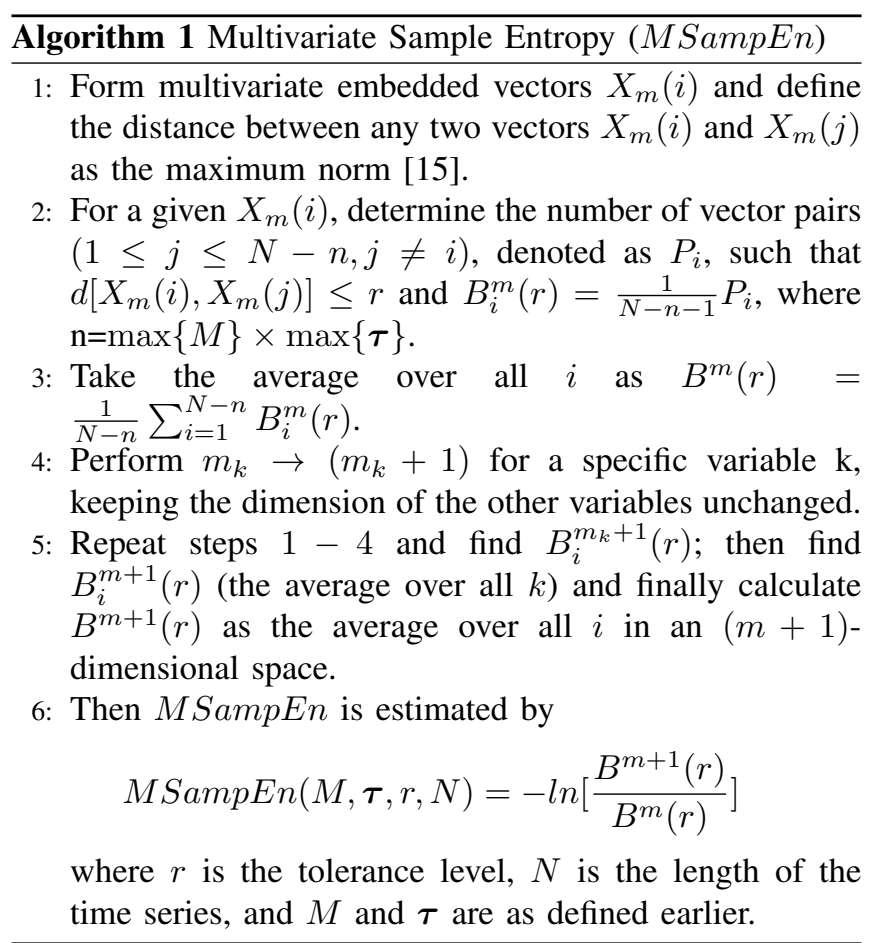

To compare the relative complexity of normalized multivariate time series, the multivariate MSE (MMSE) curves (multivariate sample entropy plotted as a function of the scale factor) are used similarly as in the univariate MSE analysis as described in [4], that is:

- A multivariate time series is considered more complex than another if for majority of the scales its entropy values are higher than other;

- A monotonic decrease of the entropy values with scale factors reveals that the signal only contains information in the smallest scale.

Using the univariate MSE analysis, it was shown in [4] that for random white noise (uncorrelated), the sample 
entropy values monotonically decrease whereas for a $1 / f$ noise (long-range correlated), the sample entropy remains constant over multiple scales. This indicates that $1 / f$ noise is structurally more complex than uncorrelated signals.

To illustrate this behaviour for the multivariate case, we generated a 5-variate time series where originally all the variables represented realisations of independent 1/f noise. We then gradually decreased the number of variables that represent 1/f noise, from 5 to 0 and simultaneously increased the number of variables that represent white noise from 0 to 5 , so that the total number of variables in the system is always 5. Fig. 1 shows the MMSE curves for these processes. Notice that as the number of variables representing 1/f noises decreases, the complexity also decreases at higher scales, and when all the channels are white Gaussian noises, the complexity is lowest at higher scales. This confirms that for multivariate processes with long range correlations, the more variables/channels have long range correlations, the higher the complexity of the whole multivariate system.

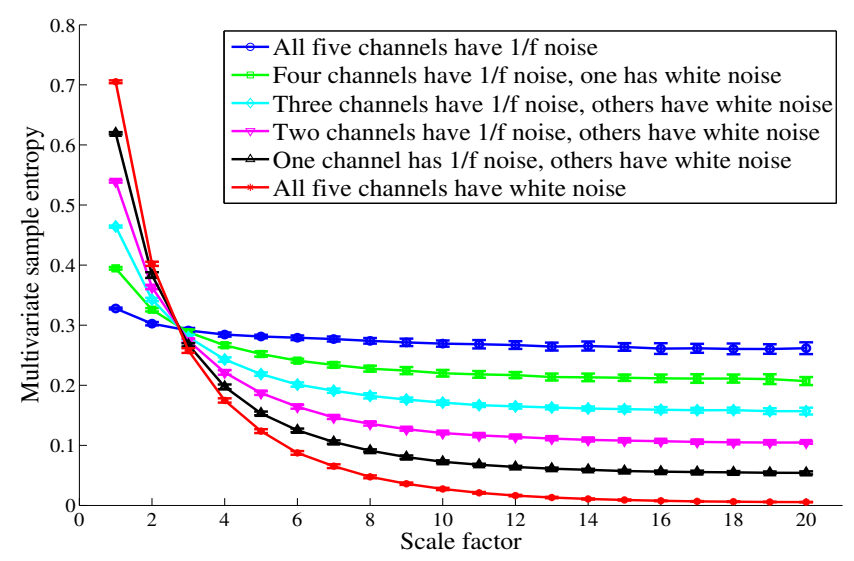

Fig. 1. Multivariate multiscale entropy analysis for multivariate white and $1 /$ f noise, each with 10,000 data points. The curves represent an average of 20 independent realizations and error bars the standard deviation (SD).

\section{BRAIN CONSCIOUSNESS APPLICATION}

In this section, we evaluated multivariate multiscale entropy (MMSE) for the characterization of brain consciousness, particularly, the coma and quasi-brain-death state. The legal definition of brain death is an irreversible loss of forebrain and brainstem functions [16], however, brain death diagnosis procedures are complicated, and some tests require temporary disconnection from medical support. An initial prognosis of quasi-brain-death (QBD) is given based on various methods used for studying brain states using electroencephalogram (EEG). Studies have shown that large activity in the alpha band reflects the alertness of a patient [17], however, standard spectral analyses are unable to yield information of the brain's inherent nonlinear complex dynamics [18], an important feature for brain states diagnosis [19]. It is natural to assume that a brain in the states of coma and quasi-brain-death would have different degrees of complexity, and that the more stressed the system the lower the complexity. As a result, methods from nonlinear dynamics theory such as MMSE are a natural choice in this context.

\section{A. Data}

The EEG data were recorded in the intensive care unit in Hua Shan Hospital, Shanghai, China using a standardized 10-20 system. The measured voltage signal was digitized via a portable EEG recording instrument with a sampling frequency of $1000 \mathrm{~Hz}$. Experimental data were obtained from 34 patients ( 16 female, 18 male) of ages ranging from 17 to 85 years old; 17 of the patients were in coma, and 17 in the quasi-brain-death (QBD) state. Unless otherwise specified, the values of the parameters used to calculate SampEn are $N=20000, m=2, \tau=1$ and $r=0.15$, chosen on the basis of previous studies indicating good statistical reproducibility [7]. For MSampEn, we used the same parameter values for every channel, though there are few methods in the literature [20] for determining the optimal embedding parameters of a signal which are beyond the scope of this paper. For MSE/MMSE, the length of each coarse-grained sequence is $\epsilon$ (scale factor) times shorter than the length of the original series, so the highest scale factor used in the analysis was 100 .

\section{B. Experimental Results}

The univariate multiscale entropy method was first applied to a single channel EEG signal (FP1) from both coma and quasi-brain-death patients. We further tested the hypothesis that the complexity of these time series is encoded in the sequential ordering of the samples of EEG signals. Therefore, for each EEG time series, we built a surrogate time series by shuffling (randomly reordering) the sequence of data points. In this way, we destroyed correlations among the data samples while preserving the statistical properties of the distribution, particularly, the first and second moments. In Fig. 2, the MSE results for EEG signals from both coma and quasi-brain-death patients and their corresponding randomly shuffled time series are presented. From the curves, for scale 1 (corresponding to single scale SampEn), physiological time series are assigned the lowest values of entropy. However, the entropy for shuffled time series monotonically decreases with an increase in the scale factor (similar to white noise), whereas the biological time series exhibitted higher entropy for larger scales as compared to the corresponding shuffled time series. Since EEG behave similarly to $1 / \mathrm{f}$ noise, these results show that biological time series are more complex than surrogate ones.

Though intuitively coma patients should have higher complexity in EEG than the quasi-brain-death patients, we cannot ascertain this from single channel MSE curves. To obtain a more comprehensive view, we applied the proposed multivariate multiscale entropy (MMSE) method over all the six electrodes (FP1, FP2, F3, F4, F7, F8). Fig. 3 shows the result where for higher MMSE scales the coma patients showed higher complexity than the QBD patients. This indicates a reduction in the intra-cortical information flow and lower 


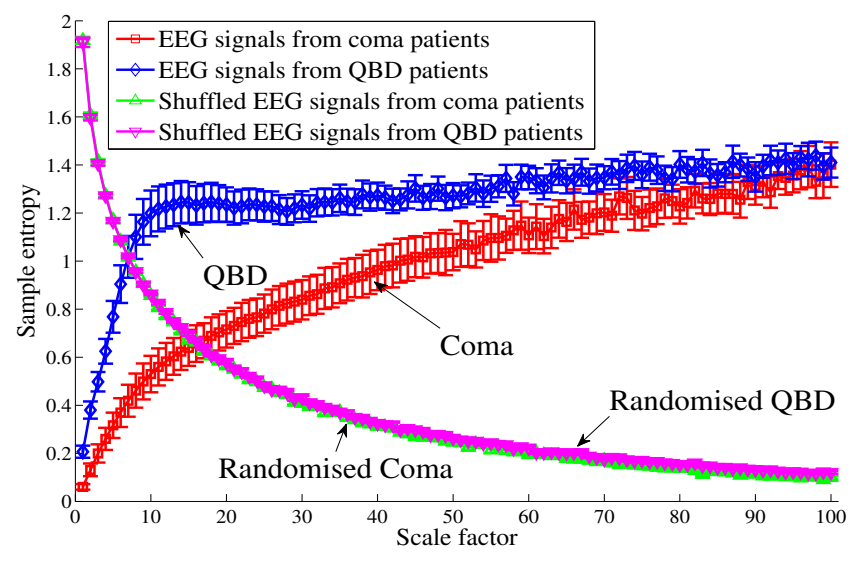

Fig. 2. Univariate multiscale entropy analysis for EEG signals from coma and QBD patients and their corresponding randomly shuffled time series.

neuronal process in the brain for the QBD patients. This also shows inactivation of previously active networks or a loss of dynamical brain responsiveness to the environmental conditions. Besides, the findings of our study support the more general concept of multiscale complexity loss with aging and disease which also reduces the adaptive capacity of biological organization at all levels [12].

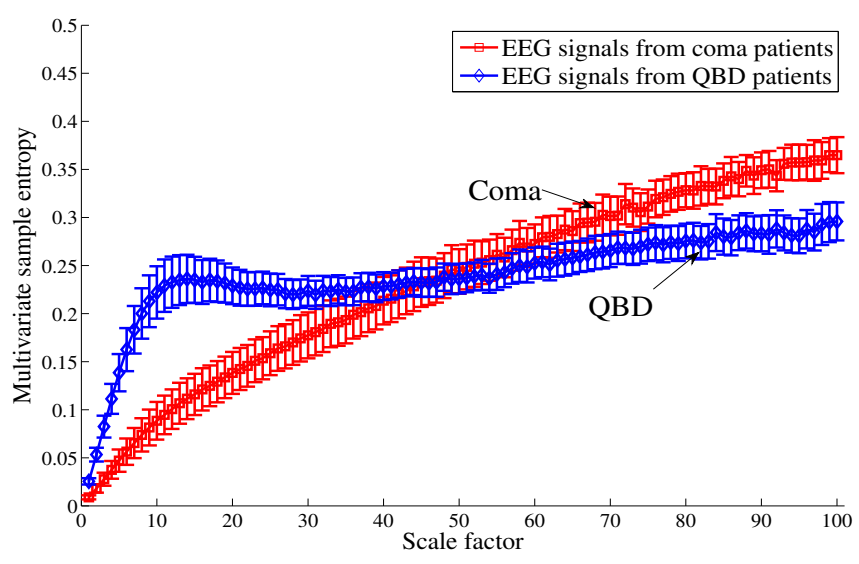

Fig. 3. Multivariate multiscale entropy analysis for EEG signals from coma and QBD patients.

\section{CONCLUSIONS}

This paper has extended the recently introduced multiscale entropy analysis method to multivariate case, as most biological systems are typically of multivariate, coupled and noisy nature. The inherent complexity of biological structures and complicated dynamics also makes multivariate multiscale entropy (MMSE) method a natural choice to reveal the long-range spatio-temporal correlations present in biological signals. The MMSE method has been applied to characterize states of brain consciousness from multichannel EEG recordings, and has shown more conclusive results than the univariate MSE method.

\section{REFERENCES}

[1] F. Takens, "Detecting strange attractors in turbulence," in Dynamical Systems and Turbulence, Warwick 1980, ser. Lecture Notes in Mathematics, D. Rand and L.-S. Young, Eds. Springer-Verlag, vol. 898, pp. 366-381.

[2] H. Kantz and T. Schreiber, Nonlinear Time Series Analysis. Cambridge, UK: Cambridge University Press, 2000.

[3] T. Gautama, D. P. Mandic, and M. M. Van Hulle, "Indications of nonlinear structures in brain electrical activity," Physical Review E, vol. 67, no. 4, p. 046204, 2003.

[4] M. Costa, A. L. Goldberger, and C. K. Peng, "Multiscale entropy analysis of complex physiologic time series," Physical Review Letters, vol. 89, no. 6, p. 068102, 2002.

[5] P. Grassberger and I. Procaccia, "Estimation of the Kolmogorov entropy from a chaotic signal," Physical Review A, vol. 28, no. 4, pp. 2591-2593, 1983.

[6] S. M. Pincus, "Approximate entropy as a measure of system complexity," Proceedings of the National Academy of Sciences of the United States of America, vol. 88, no. 6, pp. 2297-2301, 1991.

[7] J. S. Richman and J. R. Moorman, "Physiological time-series analysis using approximate entropy and sample entropy," AJP - Heart and Circulatory Physiology, vol. 278, no. 6, pp. H2039-2049, 2000.

[8] R. Hornero, D. Absolo, J. Escudero, and C. Gomez, "Nonlinear analysis of electroencephalogram and magnetoencephalogram recordings in patients with Alzheimer's disease," Philosophical Transactions of the Royal Society A: Mathematical, Physical and Engineering Sciences, vol. 367 , no. 1887 , pp. 317-336, 2009.

[9] M. Costa, C. K. Peng, A. L. Goldberger, and J. M. Hausdorff, "Multiscale entropy analysis of human gait dynamics," Physica A: Statistical Mechanics and its Applications, vol. 330, no. 1-2, pp. 5360, 2003.

[10] T. Takahashi, R. Y. Cho, T. Murata, T. Mizuno, M. Kikuchi, K. Mizukami, H. Kosaka, K. Takahashi, and Y. Wada, "Age-related variation in EEG complexity to photic stimulation: A multiscale entropy analysis," Clinical Neurophysiology, vol. 120, no. 3, pp. 476483, 2009.

[11] M. Costa, I. Ghiran, C. K. Peng, A. N. Weller, and A. L. Goldberger, "Complex dynamics of human red blood cell flickering: Alterations with in vivo aging," Physical Review E, vol. 78, no. 2, p. 020901, 2008.

[12] A. L. Goldberger, L. A. N. Amaral, J. M. Hausdorff, P. C. Ivanov, C. K. Peng, and H. E. Stanley, "Fractal dynamics in physiology: Alterations with disease and aging," Proceedings of the National Academy of Sciences of the United States of America, vol. 99, no. Suppl 1, pp. 2466-2472, 2002.

[13] J. Valencia, A. Porta, M. Vallverdu, F. Claria, R. Baranowski, E. Orlowska-Baranowska, and P. Caminal, "Refined multiscale entropy: Application to 24-h holter recordings of heart period variability in healthy and aortic stenosis subjects," IEEE Transactions on Biomedical Engineering, vol. 56, no. 9, pp. 2202 -2213, 2009.

[14] L. Cao, A. Mees, and K. Judd, "Dynamics from multivariate time series," Physica D: Nonlinear Phenomena, vol. 121, no. 1-2, pp. 75 $-88,1998$.

[15] T. Gautama, D. P. Mandic, and M. M. Van Hulle, "The delay vector variance method for detecting determinism and nonlinearity in time series," Physica D: Nonlinear Phenomena, vol. 190, no. 3-4, pp. 167176, 2004.

[16] H. K. Beecher, "A definition of irreversible coma: Report of the ad hoc committee of the Harvard medical school to examine the definition of brain death," The Journal of the American Medical Association, vol. 205, no. 6, pp. 337-340, 1968.

[17] D. P. Mandic, M. Golz, A. Kuh, D. Obradovic, and T. Tanaka, Signal Processing Techniques for Knowledge Extraction and Information Fusion. Springer, 2008.

[18] T. Gautama, D. P. Mandic, and M. M. Van Hulle, "A novel method for determining the nature of time series," IEEE Transactions on Biomedical Engineering, vol. 51, no. 5, pp. 728 -736, 2004.

[19] L. Li, Y. Xia, B. Jelfs, J. Cao, and D. P. Mandic, "Modelling of brain consciousness based on collaborative adaptive filters," presented at the International Symposium on Neural Networks, Shanghai, Chaina, Jun. 6-9, 2010.

[20] T. Gautama, D. P. Mandic, and M. M. Van Hulle, "A differential entropy based method for determining the optimal embedding parameters of a signal," in Proc. IEEE International Conference on Acoustics, Speech, and Signal Processing (ICASSP '03), vol. 6, 2003, pp. 29-32. 